\title{
Cáncer de cérvix y autorresponsabilidad: perfilando el riesgo de abstención al Papanicolaou entre las mujeres chilenas mediante Árboles de Decisión
}

\author{
Carlos Rodríguez Garcés ${ }^{1}$, Geraldo Padilla Fuentes ${ }^{1}$
}

RESUMEN

Objetivo: modelar estadísticamente los niveles de riesgo al absentismo preventivo de Papanicolaou que presentan las mujeres chilenas según variables sociodemográficas.

Materiales y métodos: Encuesta de Caracterización Socioeconómica Nacional. Muestra de 73324 casos según alcances CASEN. Modelamiento según Árboles de Decisión para establecer perfiles de la mujer que se abstiene y analizar luego los motivos que esgrimen para tal decisión.

Resultados: el riesgo de abstención al examen varía considerablemente en razón de tomar otros exámenes de prevención de cáncer (no tomar mamografía), encontrarse en determinada etapa de la adultez (tardía) y tener algún tipo de vínculo conyugal; al tradicional déficit de información y acceso le sustituyen los motivos personales como excusa frecuente para no considerar el Papanicolaou como acción recurrente. Así, la percepción de poca utilidad y la falta de tiempo configuran el comportamiento de abstención como una decisión en lugar de una imposibilidad.

Conclusiones: Tales resultados problematizan las características de la mujer contemporánea y sus comportamientos, además de plantear la necesidad de considerar la intervención socioeducativa como requisito para generar actitudes favorables hacia la prevención; factores que debiera considerar la política social de prevención del cáncer en general y de cérvix en particular.

Palabras clave: Cáncer de cuello uterino; prueba de Papanicolaou; salud de la mujer; autocuidado (Fuente: DeCS BIREME).

\section{Cervical cancer and self-responsibility: modeling the risk of refraining to undergo a papanicolaou test among Chilean women by decision trees}

\begin{abstract}
Objective: To model by statistical means the risk levels of refraining to undergo a papanicolaou test among Chilean women according to sociodemographic variables.

Materials and methods: National Socioeconomic Characterization Survey (CASEN). Sample of 73,324 cases, according to CASEN's data. Modeling by decision trees to establish the profile of women who refrain from undergoing a papanicolaou test, and then analyze the reasons for that decision.

Results: The risk of refraining to undergo a papanicolaou test varies considerably and depends on variables such as other cancer preventive tests (not undergoing a mammography), adulthood stage (late phase), and marital status. The traditional lack of information and access is replaced by personal reasons as the frequent excuse for not undergoing a papanicolaou test on a continuous basis. Perception of unusefulness and lack of time cause this refraining behavior as a decision and not as an impossibility.

Conclusions: The results question contemporary women's characteristics and behaviors, and pose the need for considering a social-educational intervention as a requirement to generate favorable attitudes towards prevention. These factors should be considered by social policy on cancer prevention in general, and particularly in cervical cancer prevention.
\end{abstract}

Keywords: Uterine cervical neoplasms; papanicolaou test; women's health; self care (Source: MeSH NLM).

1. Centro de Investigación CIDCIE, Universidad del Bío-Bío. Chillán, Chile. 


\section{INTRODUCCIÓN}

El cáncer (Ca) es una enfermedad de especial preocupación tanto desde el ámbito de la investigación biomédica como de la política social. La medicina, la bioquímica, la biología molecular, la genética y otras disciplinas de la salud, en un trabajo conjunto de aprovechamiento de las sinergias, buscan nuevos fármacos y desarrollos terapéuticos a fin de evitar el deterioro que su manifestación implica para la salud y la calidad de vida de quienes lo padecen. El Estado y organizaciones de la sociedad civil desarrollan diversas acciones para su estudio, tratamiento y prevención. El cáncer no solo se constituye en una amenaza para la vida, también constituye una catástrofe emocional y económica para los portadores, sus familiares, amigos y sociedad en general, situación que devela la importancia de la acción preventiva.

Dentro de los distintos tipos de cáncer, el de mama (CaMa) y el de cérvix $(\mathrm{CaCu})$ tienen particularidades distintivas. Ambos son los tipos de cáncer más presentes en la población femenina a nivel internacional ${ }^{(1,2)}$, cuentan con los métodos de detección más documentados y sus exámenes de detección temprana y acción preventiva tienden a ser implementados cada vez con mayor fuerza por parte de los Estados. El CaMa y $\mathrm{CaCu}$ afectan significativamente la vida de las mujeres y pueden llegar a ser mortales, en especial si evolucionan y expanden mediante metástasis ${ }^{(3)}$, no obstante sus estrategias tempranas de detección y terapia son de alta eficiencia y buen pronóstico.

En el contexto latinoamericano, el $\mathrm{CaCu}$ tiene una alta prevalencia, particularmente debido a los déficits de cobertura del control epidemiológico, la escasa disponibilidad de recursos, el acceso segmentado a los servicios de salud y la baja instrucción en prevención primaria que tiene la población ${ }^{(4)}$.

Aun cuando a nivel general los Estados han ofrecido planes de tamizaje que incluyen diagnósticos gratuitos y aseguran atención en casos de alto riesgo, en última instancia es la decisión personal de las mujeres la que define el éxito en el control del cáncer. En lo que a $\mathrm{CaCu}$ repecta, más allá de la disponibilidad de medios, la acción preventiva requiere de una importante toma de conciencia respecto de su necesidad y objetivos, percepción que varía en razón de los niveles de desarrollo y distribución de riquezas que exhiben los países de la región ${ }^{(5,6)}$.

Mientras por un lado se discute la confiabilidad, potencialidades, déficits y precisión de los métodos de detección de estas enfermedades (prevención primaria), por otro se hace hincapié en la necesaria educación, sensibilización y concientizacion de la población en torno a la acción preventiva (prevención secundaria), reduciendo la exposición a factores de riesgo y anticipando la detección oportuna, lo que en ocasiones puede resultar tan eficiente como los exámenes médicos que lo corroboran ${ }^{(7,8)}$. El conjunto de comportamientos que pone en práctica de manera cotidiana y consciente el sujeto definen un estilo de vida que puede posicionarlo en una situación de riesgo para con la enfermedad. El autocontrol, las acciones de autocuidado o de autorresponsabilidad con la salud son mecanismos comportamentales que permiten no solo realizar cambios hacia estilos de vida más saludables, sino que además preservar su buena salud evitando conductas de riesgo, pero esta acción preventiva estará fuertemente modelada por la motivación como elemento estructurante en la regulación de la conducta ${ }^{(9)}$.

La situación en Chile, en cuanto a acción preventiva y no obstante sus especificidades, es un correlato del acontecer internacional. Según cifras, al año 2011 la abstinencia a la mamografía en Chile alcanzaba el 50,2 \% en mujeres mayores de 35 años, y un 42,1\% en el caso del Papanicolaou para aquellas sobre 15 años ${ }^{(10)}$. En mediciones recientes la situación no ha cambiado considerablemente, con registros de abstención alrededor del 38,2 \% para Papanicolaou y 40,7 $\%$ para mamografías ${ }^{(11)}$. Las explicaciones a estos déficits de cobertura dan cuenta de la multidimensionalidad transversalizada del fenómeno, el cual no solo reúne motivos económicos o de disponibilidad, sino que también alcanza las nuevas características en que se desenvuelve la mujer, las demandas que enfrenta y sus intereses, además de la valoración y percepción de riesgo que tiene frente a la probabilidad de padecimiento. No se trata solo del examen en sí, ya que ambos han probado ser eficaces, atenuantes y dúctiles ${ }^{(12-15)}$, sino también de considerar las persistentes trabas de desinformación, vulnerabilidad social, acceso restringido a los servicios de salud, situación laboral, baja escolaridad, acceso y/o precariedad del servicio disponible, condición de afiliación a la seguridad social; además de los efectos que el temor y la duda tienen en las mujeres para evitar someterse a dicho examen ${ }^{(16-23)}$.

En suma, resulta de interés para este artículo indagar respecto del comportamiento de la abstención, definiendo perfiles y trayectorias de riesgo en la toma de decisión frente a la acción preventiva, bajo el supuesto que la autorresponsabilidad y el autocuidado trascienden el marco institucional y de la política social para irrumpir en componentes educativos y culturales del sujeto quien asume conductas muchas veces incompatibles con la buena salud. Para ello hace uso de la base de datos de la Encuesta de Caracterización Socioeconómica Nacional aplicada en Chile el año 2015, modelando la técnica multivariante de Árboles de Decisión. Metodología de análisis que si bien no es muy común en estos ámbitos investigativos, se perfila como una herramienta útil y confiable en la identificación de patrones de comportamiento y grupos poblacionales de riesgo.

La ventaja que presenta este trabajo en relación a lo revisado, es que hace uso de los datos recientemente publicados de la encuesta CASEN 2015, los cuales son representativos de la situación nacional y permiten alcanzar inferencias en un grado de alta confiabilidad. Bajo el supuesto de que las trabas tradicionales han sido relativamente desplazadas por nuevas dificultades, se presenta un análisis de regresión y plantean determinados factores de riesgo asociados a la abstención frente a la acción preventiva. 


\section{MATERIALES Y MÉTODOS}

Instrumento

Mediante el uso de la base de datos de la Encuesta de Caracterización Socioeconómica Nacional (CASEN) en su reciente edición 2015, se analizan los factores que inciden en los índices de abstención al examen de Papanicolaou, asi como los motivos reportados por las mujeres chilenas para no ser partícipes de esta acción preventiva con base a sus perfiles de riesgo.

La CASEN es un instrumento de recolección de datos de carácter oficial, cuya validez métrica y poblacional le permite constituirse en el principal instrumento de diagnóstico de la realidad nacional e insumo para la elaboración y ajustes de la política social en Chile, como lo serían las vinculadas con salud preventiva.

\section{Muestra}

La encuesta CASEN obtiene muestras de representación nacional mediante procedimientos estratificados $y$ polietápicos. Para efectos de este estudio se consideran los datos a nivel nacional para el segmento de mujeres mayores de 35 años. En términos de atributos sociodemográficos, estas se caracterizan por habitar en zonas urbanas $(78,1 \%)$, tener pareja (56,9\%), ser menor de 55 años (53,0\%), encontrarse laboralmente activas (40,9\%), y en su gran mayoría tener educación secundaria, generalmente completa, donde solo un $16,8 \%$ señala cursar o haber cursado estudios superiores completos o incompletos (Tabla 1).

Tabla 1. Caracterización de la muestra $(n=73.324)$

\begin{tabular}{ll} 
Rango etario & \\
- Entre 35 y 55 años & 53,0 \\
- 56 y más años & 47,0 \\
\hline Situación conyugal & \\
- Con pareja & 56,9 \\
- Sin pareja & 43,1 \\
\hline Situación ocupacional & \\
\hline -Ocupada & 40,9 \\
-Desocupada & 2,4 \\
-Inactiva & 56,7 \\
\hline Escolaridad & \\
\hline -Hasta 8 años & 37,9 \\
-Entre 9 y 12 años & 41,0 \\
-Más de 12 años & 16,8 \\
\hline Zona & \\
\hline -Urbana & 78,1 \\
-Rural & 21,9
\end{tabular}

Fuente: CASEN 2015. Elaboración propia

\section{Procedimiento}

En primera instancia, se modelan los factores que inciden en la acción preventiva mediante arboles de decisión con base al comportamiento de un conjunto de variables teóricamente relevantes. Este algoritmo de árboles de clasificación permite una representación gráfica intuitiva para grandes cantidades de datos con alto nivel de confiabilidad por medio de una estrategia sistemática al observar las variables más relevantes de forma escalonada y proporcionar reglas que operan sobre las decisiones tomadas. Su capacidad de discriminación multivariante maximiza la distancia entre grupos, y entrega nodos (variables de entrada), ramas (subcategorías de variables vinculadas) y hojas (valores de las subcategorías) que, junto con permitir una clara interpretación y visualización, define y valida modelos con capacidad predictiva ${ }^{(15,24-26)}$, otorgando la posibilidad de estimar atributos cualitativos como la decisión de abstención en las mujeres. Ello posibilita, junto con resumir grandes volúmenes de datos, encontrar relaciones insospechadas entre las variables y definir perfiles, los cuales son útiles para definir los lineamientos en política social de salud preventiva.

El método de segmentación utilizado para el desarrollo del árbol de decisión fue Chi-square Automatic Interaction Detection (CHAID), basado en chi2 para seleccionar a los predictores. La variable criterio, dado el carácter restrospectivo del estudio, corresponde a la decisión de realizarse o no el Papanicolaou en los últimos 3 años. Variable de expresión dicotómica (sí;no) que busca caracterizar el riesgo de marginación a la acción preventiva 
Cáncer de cérvix y autorresponsabilidad: perfilando el riesgo de abstención al Papanicolaou entre las mujeres chilenas mediante Árboles de Decisión

modelada que presentan las mujeres. Como variables predictoras se utilizaron edad, escolaridad, tenencia de pareja, situación de afiliación, grupo socioeconómico, screening mamario, zona de residencia, condición de actividad y pobreza, cuya operacionalización (Tabla 2).

Tabla 2. Variables incluidas en la modelación de la abstención al Papanicolaou

\begin{tabular}{|c|c|c|}
\hline Variable & $\begin{array}{l}\text { Operacionalización (categorías que } \\
\text { comprende) }\end{array}$ & $\begin{array}{l}\text { Situación } \\
\text { [Simetrías] }\end{array}$ \\
\hline Edad & $\begin{array}{l}\text { Medida en rangos etarios según ciclo vital de } \\
\text { las mujeres (35-54 años; mayor de } 54 \text { años) }\end{array}$ & $\begin{array}{l}\text { Significativa, segundo } \\
\text { nivel jerárquico. } \\
{[35-54=0,53 ;>54=0,47]}\end{array}$ \\
\hline Escolaridad & $\begin{array}{l}\text { Categorías que son expresión de los años de } \\
\text { asistencia a educación formal (Hasta } 8 \text { años; } \\
\text { entre } 9 \text { y } 12 \text { años; más de } 12 \text { años) }\end{array}$ & $\begin{array}{l}\text { No significativa } \\
\begin{array}{c}{[<9=0,42 ; 9-12=0,41} \\
\quad>12=0,17]\end{array}\end{array}$ \\
\hline Tenencia de pareja & $\begin{array}{c}\text { Situación sentimental de la mujer, definida } \\
\text { según la existencia o no de un cónyuge } \\
\text { independiente de su sexo (con pareja; sin } \\
\text { pareja). }\end{array}$ & $\begin{array}{l}\text { Significativa, tercer nivel } \\
\text { jerárquico. } \\
\text { [Sí=0,57; No=0,43] }\end{array}$ \\
\hline Sistema de afiliación & $\begin{array}{l}\text { Adscripición a sistema de salud } \\
\text { complementario al de asistencia social básica } \\
\text { (Público; privado; ninguno) }\end{array}$ & $\begin{array}{c}\text { No significativa } \\
\text { [Privado }=0,12 ; \text { Público }= \\
0,86 ; \text { Ninguno }=0,02] .\end{array}$ \\
\hline Grupo socieconómico & $\begin{array}{l}\text { Agrupación según quintil de ingresos per cápita } \\
\text { nacional del hogar (I; II; III; IV; V). }\end{array}$ & $\begin{array}{c}\text { No significativa } \\
{[l=0,24 ; \mathrm{ll}=0.23 ; \mathrm{ll}=} \\
0,21 ; \mathrm{IV}=0,18 ; \mathrm{V}=0,14]\end{array}$ \\
\hline Realización mamografía & $\begin{array}{l}\text { Realización de examen preventivo } \\
\text { mamográfico en los últimos } 3 \text { años (sí; no). }\end{array}$ & $\begin{array}{l}\text { Significativa, primer nivel } \\
\text { jerárquico. } \\
\text { [Sí=0,58; } \mathrm{No}=0,42]\end{array}$ \\
\hline Zona & Lugar de residencia (urbano; rural). & $\begin{array}{c}\text { No significativa } \\
\text { [Urbano= } 0,78 ; \text { Rural }= \\
0,22] .\end{array}$ \\
\hline Condición de actividad & $\begin{array}{l}\text { Situación laboral de la mujer en el último mes } \\
\text { (Ocupada; desocupada/Inactiva). }\end{array}$ & $\begin{array}{c}\text { No significativa } \\
\text { (Ocupada }=0,41 ; \\
\text { Desocupada/Inactiva }= \\
0,59] .\end{array}$ \\
\hline Pobreza multidimensional & $\begin{array}{c}\text { Estado de pobreza según enfonque } \\
\text { multidimensional, es decir, en sintonía con la } \\
\text { vulnerabilidad familiar en educación, trabajo, } \\
\text { ingresos, salud y previsión social (Pobre; no } \\
\text { pobre). }\end{array}$ & $\begin{array}{l}\text { No significativa. } \\
\text { [Pobre }=0,21 ; \text { No pobre }= \\
0,79]\end{array}$ \\
\hline Realización Papanicolaou & $\begin{array}{c}\text { Realización de examen preventivo CaCu en los } \\
\text { últimos } 3 \text { años (si; no). }\end{array}$ & $\begin{array}{l}\text { Variable modelada. } \\
{[S i ́=0,68 ; \mathrm{No}=0,32]}\end{array}$ \\
\hline
\end{tabular}


El análisis exploratorio de las variables contempladas inicialmente en el modelo, tanto significativas como no significativas, muestra adecuadas asimetrías de tolerancia, tanto para la variable dependiente como las independientes, verificándose el cumplimiento de la regla “90/10" en razón de su naturaleza categorial. Con esto se verifican los criterios de asimetría recomendados para la aplicación de modelos que involucren el cálculo de jicuadrados usados en árboles de decisión, dado el rango de 0,12 y 0,86 dentro del que se sitúan los valores de las categorías, cifras que corresponden a la adscripción pública o privada a los sistemas de salud complementarios respectivamente.

Para la confección del árbol, dado el tamaño muestral que proporciona la CASEN, y a fin de simplificar la relevancia jerárquica de las ramas y cuantía de los nodos se optó por solicitar un árbol de 3 niveles con podas cuando no se superaran los 1000 casos para el nodo padre y 500 para el nodo hijo, a fin de evitar el sobreajuste del modelo. Exigencias para el algoritmo de clasficación que terminan por configurar un árbol de decisión conformado por 3 niveles de profundidad, 15 nodos, 9 de ellos terminales. El nodo más pequeño, una vez excluido el nodo 3 que contempla los valores perdidos del screening, agrupa el $7,8 \%$ de los casos de la muestra.

Para apoyar la significancia entre nodos jerarquizados expuesta por ji cuadrado se calcularon las fuerzas o pesos de las relaciones con base a los coeficientes de asociación Phi y $\mathrm{V}$ de Cramer según condiciones o grados de libertad de la tabla de contingencia resultante.

La estimación de riesgo alcanza un $18 \%$, en otras palabras, a nivel general el $82 \%$ de los casos es clasificado por el modelo en la misma categoría observada en la muestra. En términos específicos, en la categoría abstención al examen Papanicolaou (categoría objetivo) califica correctamente solo al 54,8 \% de los casos, aunque al considerar la categoría que representa a las mujeres que sí se han efectuado el examen los casos corectamente clasificados ascienden al 94,8 \%.

Tabla 3. Definición operacional de categorías motivacionales

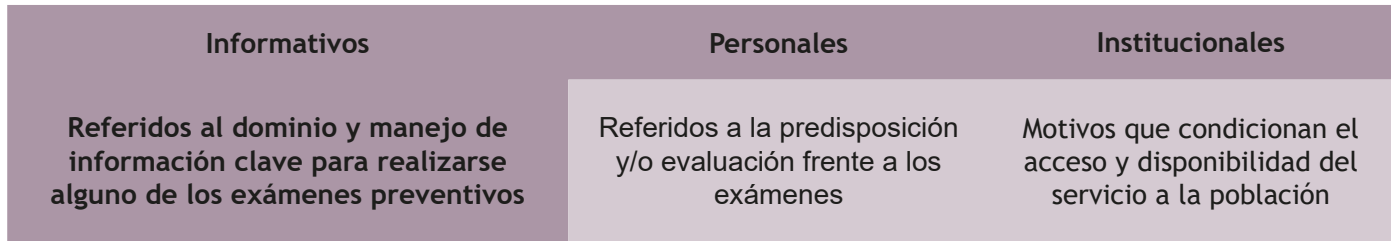

En una segunda parte se hace un análisis descriptivo y de corte comparativo de los motivos reportados por las mujeres para no realizarse el examen, los cuales se agrupan según su eje temático en tres categorías (informativo, personal e institucional) y son analizados tanto a nivel general como sobre la base de los perfiles de riesgo generados a partir del modelamiento resultante del árbol de decisiones.

\section{DISCUSIÓN}

Del universo de mujeres de 35 años y más, un $32,1 \%$ no se ha realizado el examen de Papanicolaou y un $41,6 \%$ se ha abstenido también de efectuarse el screening mamográfico.

Al momento de ilustrar mediante árboles de clasificación (Figura 1) el perfil demográfico de las mujeres que se abstienen de realizar el examen preventivo para $\mathrm{CaCu}$, se observa que el factor de mayor capacidad de segmentación es la opción de autocuidado vinculada con el screening mamario [X2 $(2)=21417,109, \mathrm{p}<, 01]$, cuya fuerza de asociación es moderadamente intensa [ $\mathrm{Vc}=, 555 ; \mathrm{p}<, 01]$. De entre quienes se abstienen de realizar el control preventivo mamográfico, un $62,8 \%$ se excluye también de la acción preventiva para $\mathrm{CaCu}$, mientras que cuando declaran habérselo realizado esta cifra de abstención baja a un $10,3 \%$. Con esto se puede defender la concomitancia en la integralidad de las decisiones de autocuidado, por cuanto el someterse a un tipo de examen aumentaría la probabilidad de tomar igual decisión respecto de otro, y viceversa. Es así como del 32,1\% que inicialmente declara automarginarse de la acción preventiva del Papanicolaou (nodo 0), esta proporción disminuye al 10,3\% al considerar el nodo de las mujeres que si se han realizado el screening (nodo 1), lo que representa una reducción del 0,68 de probabilidad. De igual manera, esta probabilidad de exclusión se ve casi duplicada, aumentando en un 95 $\%$, en el segmento de mujeres que declaran no haberse efectuado la mamografía (nodo 2). 
Cáncer de cérvix y autorresponsabilidad: perfilando el riesgo de abstención al Papanicolaou entre las mujeres chilenas mediante Árboles de Decisión

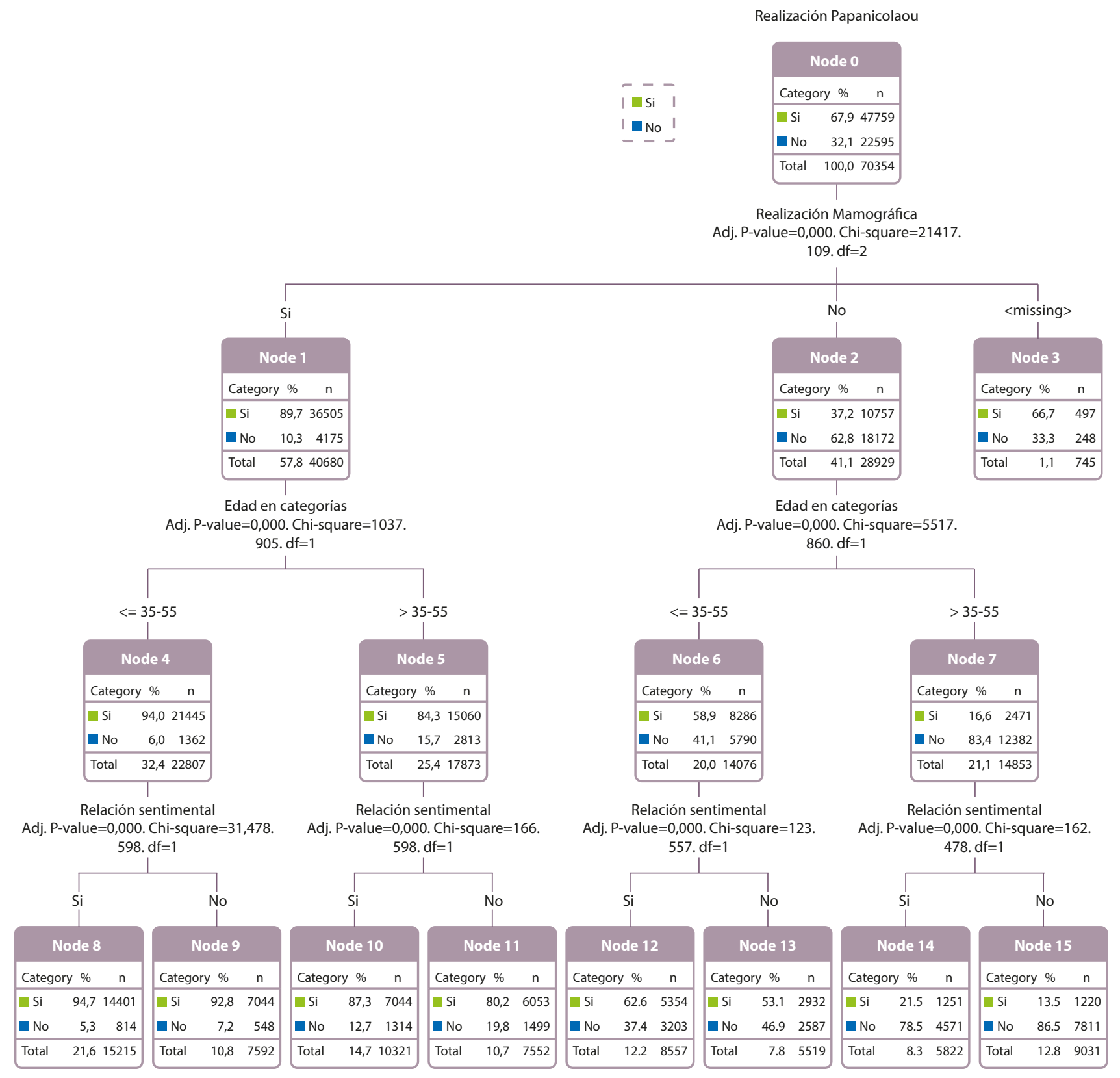

Figura 1. Árbol de decisiones respecto de la abstención al Papanicolaou

En un segundo nivel de clasificación jerárquica y para ambos grupos derivados del screening, la edad o rango etario se configura como la variable de mayor relevancia discriminativa, aunque con un comportamiento de riesgo diferenciado. Para el nodo 1, que representa al segmento de mujeres que declara haberse efectuado este test, la capacidad protectora de la acción preventiva concomitante se ve influenciada por el rango etario [X2 (1) $=1037,905, \mathrm{p}<, 01 ; \mathrm{Vc}=, 160, \mathrm{p}<, 01]$, de modo que cuando tienen menos de 55 años (nodo 4) la proporción de quienes no se ha realizado el Papanicolaou baja al $6 \%$, mientras que en el nodo 5 (>55 años), asciende a un 15,7\%.

Por su parte, respecto de quienes no se han realizado el screening (nodo 2), la mayor edad aumenta igualmente el riesgo de abstención [X2 (1) $=5517,860, p<, 01]$, con una intensidad moderada $[\mathrm{V} c=, 437, \mathrm{p}<, 01]$. Es así como, cuando las mujeres tienen más de 55 años y no se han efectuado el screening (nodo 7), la probabilidad de abstención aumenta en un 32,1\%, informando que 8 de cada 10 mujeres $(83,4 \%)$ pertenecientes a este nodo se auto-excluyen también del Papanicolaou. En consecuencia, 
la mayor edad se constituye en un factor de riesgo frente a la acción preventiva modelada, aunque su incidencia tendrá un comportamiento diferencial según las elecciones previamente tomadas respecto de otros comportamientos de autorresponsabilidad frente al cáncer, como lo es el examen de mamografía.

La situación conyugal de las mujeres, expresada en el hecho de tener o no pareja con independencia de la naturaleza del vínculo, aparece como la tercera variable con capacidad discriminativa frente a la conducta autorresponsable en salud modelada. En este nivel de segmentación, tenencia de pareja se constituye en una condición que disminuye la probabilidad de excluirse a la acción preventiva predicha. Así por ejemplo, para las mujeres que pertenecen al conglomerado etario mayor de 55 años y no se han realizado el screening mamario la ausencia de pareja aumenta significativamente el riesgo de abstención [X2 (1) = 162,478, $\mathrm{p}<, 01]$, el cual se posiciona en un 0,87 (nodo 15). Por otro lado, cuando se han realizado la mamografía, son menores de 55 años y cuentan con cónyuge, el riesgo de marginación que tienen las mujeres disminuye $[X 2(1)=31,478, p<, 01]$, alcanzando un escaso $5,3 \%$ (nodo 8 ).

En su conjunto el árbol de segmentación da cuenta de 9 nodos terminales. Todos ellos configurados con base a los atributos jerárquicos de mamografía, rango etario y tenencia de pareja. Tomando en consideración que dentro de la variable a modelar la categoría objetivo era la abstención frente a la acción preventiva del Papanicolaou, los perfiles de mujeres de mayor y menor riesgo vienen determinados por:

1. Alto riesgo: mujeres que no se han efectuado el examen preventivo para CaMa, están en etapas de adultez tardía (55 años y más) y no cuentan con pareja. Entre las mujeres que reúnen estas características, el riesgo asociado a la omisión de examen Papanicolaou es de 0,87 . Cuando declaran tener cónyuge, manteniendo los otros atributos constantes, el riesgo es 0.79 .

2. Bajo riesgo: constituyen factores protectores frente a la acción preventiva copulativa y jerárquicamente realizarse el examen mamográfico, tener menos de 55 años y disponer de cónyuge. Para este perfil de mujeres el riesgo de autoexclusión es de solo 0,05. Cabe señalar que el peso discriminatorio de la tenencia de pareja en el grupo de mujeres que se han hecho un screening y tienen menos de 55 años es menor pero estadísticamente significativo, aumentando el riesgo a un 0,07 .

Una vez conocidos los factores de mayor capacidad discriminativa respecto de la acción preventiva, así como los perfiles que caracterizan la abstención de las mujeres con alto riesgo (no se han realizado mamografía/ tienen más de 55 años/ no cuentan con pareja) y bajo riesgo (sí se han realizado screening mamario/ tienen menos de 55 años/ mantienen una relación conyugal), interesa conocer la naturaleza de los motivos que esgrimen estas distintas tipologías de usuaria para excluirse de la acción preventiva que constituye el examen del Papanicolaou (Tabla 4).

Tabla 4. Razones que esgrimen las mujeres que omiten Papanicolaou según perfiles de riesgo (\%)

\begin{tabular}{|lccc}
\hline & Bajo & Alto & General \\
\hline Información & & & \\
\hline - No sabe dónde hacérselo & 0,7 & 0,8 & 0,7 \\
\hline - No sabía que tenía que hacerse ese examen & 1,2 & 3,9 & 2,4 \\
\hline - No conoce ese examen & 0,2 & 1,7 & 0,9 \\
\hline Personales & & & \\
\hline - Le da miedo o le disgusta & 13,1 & 14,5 & 14,9 \\
\hline - Se le olvida hacérselo & 26,6 & 10,5 & 18,9 \\
\hline - No cree que la necesite & 18,0 & 55,7 & 37,8 \\
\hline - No tiene tiempo & 28,8 & 7,5 & 16,4 \\
\hline - No tiene dinero & 0,7 & 1,2 & 1,6 \\
\hline Institucional & & & 1,2 \\
\hline - El horario del consultorio no le sirve & 2,0 & 0,6 & 5,2 \\
\hline - No ha podido conseguir hora & 8,8 & 3,6 & \\
\hline
\end{tabular}


En general, las tipologías motivacionales alcanzan una amplia gama de manifestaciones, siendo las de naturaleza personal las más recurrentes, 9 de cada 10 mujeres $(89,6 \%)$ exponen razones de esta índole.

Los déficit institucionales e informacionales son de presencia mucho más marginal. Contrastadas las motivaciones para abstenerse con base a la tipología de riesgo proporcionada por el árbol de segmentación se perfilan diferencias relevantes. Si bien en ambos segmentos las razones de índole personal siguen siendo importantes, al desagregar se observa la predominancia que adquiere la tipología específica asociada a la percepción de inutilidad y/o incredulidad respecto de su eficacia y beneficios. En efecto, la opción "no cree que la necesite" es planteada por 1 de cada 2 mujeres que pertenecen al perfil de alto riesgo de abstención (55,7 \%), misma opción que es esgrimida solo por el $18 \%$ de las mujeres de bajo riesgo, donde la disponibilidad de tiempo $(28,8 \%)$ o el olvido $(26,6 \%)$ son las más relevantes. Aunque las motivaciones que atienden a déficit informacionales y/o institucionales son menos frecuentes, se observa una relativa mayor recurrencia de las razones vinculadas a la carencia de información en las mujeres de alto riesgo, mientras que los hándicaps institucionales lo son para las de bajo riesgo.

En el contexto nacional parece ser que las vicisitudes de la vida diaria y las singularidades de la mujer, en particular su perfil actitudinal que define la percepción subjetiva de costo/beneficio, las que definen en última instancia la toma de decisión hacia la acción preventiva. Atributos basales de la mujer pueden constituirse en factores de riesgo, tales como lo son la edad, la tenencia de pareja y las decisiones de autocuidado tomadas con ocasión de otros exámenes de igual naturaleza. Perfil de riesgo que es complementado por componentes actitudinales. Nos referimos aquí a las apreciaciones de carácter subjetivo respecto de la valoración, pertinencia y eficacia que tienen los exámenes preventivos a juicio de las eventuales usuarias.

En consecuencia, el mejoramiento de los niveles de cobertura que alcanza la acción preventiva frente al cáncer no está dada única y exclusivamente por la mejora de los niveles de acceso a la infomación o superación de los déficit de cobertura mejorando equipamiento, oportunidad, accesibilidad y calidad de los servicios de salud, sino también por la consideración de estrategias socioeducativasque procuren al conformación de un perfil más favorable hacia la acción preventiva. Campañas públicas de marketing social (27) que promuevan la aceptación y adscripción a programas que mejoren su propio bienestar y el del grupo social al que pertenecen, a la vez que rechacen o abandonen conductas sociales de riesgo para con su salud.

En conclusión, como enfermedad, el cáncer extiende sus perjuicios desde las dimensiones personales hacia las relacionales, además de poner en peligro la vida y deteriorar la salud, altera el estado de bienestar social y económico. Dentro del contingente femenino el cáncer de mamas y de cérvix son los de mayor prevalencia epidemiológica y mortalidad, ello a pesar de ser también los de mejor perspectiva de tratamiento en situaciones de detección temprana y contar con exámenes preventivos de probada eficacia. En especial, el cáncer de cérvix se ha instalado como objeto de una serie de procedimientos y exámenes tendientes a su prevención, detección y tratamiento, no obstante ello el nivel de adscripción a la acción preventiva es bajo. Atendiendo a esto, este artículo expuso mediante Árboles de Decisión los perfiles de riesgo e itinerarios que congifuran la toma de decisión respecto de la abstención al Papanicolaou por parte de mujeres mayores de 35 años, así como los motivos personales, instuticionales e informacionales que esgrimen.

Como resultado del modelamiento multivariante del árbol de decisión se estructuraron como significativas y en términos jerárquicos las variables realización de mamografía, rango etario y tenencia de pareja. Otras variables incorporadas al modelo, tales como escolaridad, zona de residencia y condición ocupacional no registraron significancia o peso relevante para el modelo.El Árbol de Decisión configura en consecuencia un total de 3 niveles con 15 nodos, 9 de ellos terminales.

Del modelamiento decisional con base al criterio objetivo (abstención), se erigieron los perfiles de bajo y alto riesgo. El perfil de bajo riesgo se define conforme a la acción de autorresponsabilidad expresada en la realización de exámenes preventivos complementarios como lo es la mamografía para CaMa, además de encontrarse en adultez temprana o media, y mantener una relación conyugal. Por el contrario, los componentes que perfilan una mayor probabilidad de riesgo son la omisión de screening mamario, ser mayor de 55 años y no mantener un vínculo conyugal.

Para ambos perfiles, los motivos de abstención son de naturaleza personal, alcanzando una hegemonía muy por sobre los asociados a déficit informacionales 0 institucionales. Dentro de las razones personales para autoexcluirse de la acción preventiva en las mujeres con perfil de alto riesgo destacan componentes actitudinales vinculados con las valoraciones respecto de la utilidad, pertinencia y eficacia del Papanicolaou.

La relevancia que alcanza el screening mamario en el modelamiento de la abstención configura la concomitancia de las decisiones vinculadas al autocuidado o autorresponsabilidad en salud. Por otra parte, la preponderancia de los motivos personales, en particular las percepciones negativas que el mythos vehicula y deposita en el imaginario femenino, obstaculizan la toma de decisión respecto de la acción preventiva en paulatina sustitución de las tradicionales categorías de déficit de información y cobertura.

En consecuencia, el objetivo de las actuales políticas sanitarias debería ser la instauración de una cultura preventiva que tribute hacia la actitud autorresponsable y evite conductas de riesgo en salud, mejorando sus 
estrategias de comunicación sobre los riesgos de padecimiento del cáncer y la eficacia de sus instrumentos de detección temprana, como lo es el examen del Papanicolaou.

\section{REFERENCIAS BIBLIOGRÁFICAS}

1. Robles SC, Galanis E. El cáncer de mama en América Latina y el Caribe. Rev Panam Salud Pública. 2002;12(2):141-3. Disponible en: https://scielosp.org/article/rpsp/2002. v12n2/141-143/es/

2. Ferlay J, Shin HR, Bray F, Forman D, Mathers C, Parkin DM. Estimates of worldwide burden of cancer in 2008: GLOBOCAN 2008. Int J Cancer. 2010;127(12):2893-917.Disponible en: https: / / www.ncbi.nlm.nih.gov/pubmed/21351269

3. Piñeros $M$, Sánchez $R$, Cendales $R$, Perry $F$, Ocampo $R$, García ÓA. Características sociodemográficas, clínicas y de la atención de mujeres con cáncer de mama en Bogotá. Rev colomb cancerol. 2008;12(4):181-90. Disponible en: http://www.cancer.gov.co/images/revistas/2008/ volumen4/3.\%20Caracteristicas\%20sociodemograficas.pdf

4. Vidal C, Hoffmeister L, Biagini L. Tendencia de la mortalidad por cáncer de cuello uterino en Chile: aplicación de modelos de regresión joinpoint.. Rev Panam Salud Pública, 2013; 33(6): 407-13. Disponible en: https://scielosp.org/ $\mathrm{pdf} / \mathrm{rpsp} / 2013 . \mathrm{v33n6/407-413/es}$

5. Programa de Cancer. Mejor detección, tratamiento y vacunas asequibles para prevenir muertes por cancer cervicouterino [Internet].Mexico: Organización Mundial de la Salud, Organización Panamerica de Salud; 2009. Disponible en: http://www.paho.org/hq/index.php?option=com_co ntent\&view $=$ article\&id $=142 \% 3$ A2008- - better -screening affordable-vaccines-prevent-cervical-cancer-deaths\&catid $=1872 \% 3$ Acancer\&ltemid $=40602 \&$ lang $=$ es

6. Arzuaga-Salazar MA, de Souza MdL, de Azevedo Lima VL. El cáncer de cuello de útero: un problema social mundial. Rev Cubana Enfermer. 2012;28(1):63-73. Disponible en: http://scielo.sld.cu/scielo.php?script=sci_ arttext\&pid=S0864-03192012000100007

7. Ponce M. La prevención del cáncer de cuello de útero y de mama en servicios de salud y organizaciones no gubernamentales de la Ciudad Autónoma de Buenos Aires. Salud colect. 2013;9(2):215-33. Disponible en: $\quad$ http://www.scielo.org.ar/scielo.php?script=sci_ arttext\&pid $=$ S1851-82652013000200007

8. FicaA. Prevención del cáncer cérvico-uterino en Chile: mucha vacuna y poco Papanicolau. Rev chil Infectol. 2014;31(2):196203. Disponible en: http://www.scielo.cl/scielo. php?script=sci_arttext\&pid=S0034-98872009000700021

9. Cañero AH. Mortalidad por cardiopatía isquémica en Cuba. Relación con la dieta y el colesterol sérico. Rev Cuba Cardiol Cir Cardiovasc. 1999;13(1): 8-12. Disponible en: http: / /bvs.sld.cu/revistas/car/vol13_1_99/car02199.pdf

10. Observatorio Social. Encuesta CASEN, 2011[Internet].Chile: Ministerio de Desarrollo Social, Gobierno de Chile, 2011. Disponible en: www.ministeriodesarrollosocial.gob.cl/ observatorio/casen/.

11. Observatorio Social. Encuesta CASEN, 2015[Internet].Chile: Ministerio de Desarrollo Social, Gobierno de Chile, 2015. Disponible en www.ministeriodesarrollosocial.gob.cl/ observatorio/casen/.
12. Tabar L, Fagerberg G, Duffy S, Day N, Gad A, Gröntoft O. Update of the Swedish two-county program of mammographic screening for breast cancer. Radiologic Clinics of North America. 1992;30(1):187-210. Disponible en: https://www.ncbi.nlm.nih.gov/pubmed/1732926

13. Camacho J. Screening mamario. Medwave. 2001;1(1):e2292. Disponible en: http://www.medwave.cl/link.cgi/ Medwave/Reuniones/per1/2292

14. Uchida M. Mamografía de screening y realidad chilena. Rev Chil Radiol. 2008;14(3):130-4.Disponible en: $\quad$ https: //scielo. conicyt.cl/scielo.php?pid=S071793082008000300005\&script=sci_arttext

15. López-Carrillo L, Suárez-López L, Torres-Sánchez L. Detección del cáncer de mama en México: síntesis de los resultados de la Encuesta Nacional de Salud Reproductiva. Salud pública Méx. 2009;51(2):s345-s9.Disponible en: http: / / www.scielo.org.mx/scielo.php?pid=S003636342009000800027\&script=sci_arttext

16. Aguilar-Pérez JA, Leyva-López AG, Angulo-Nájera D, Salinas A, Lazcano-Ponce EC. Tamizaje en cáncer cervical: conocimiento de la utilidad y uso de citología cervical en México. Rev Saude Publica. 2003;37(1):100-6. Disponible en: http://www.scielo.br/pdf/rsp/v37n1/13550

17. Lucumí Cuesta DI, Gómez Gutiérrez LF. Accesibilidad a los servicios de salud en la práctica de citología reciente de cuello uterino en una zona urbana de Colombia. Rev Esp Salud Pública. 2004;78(3):367-77. Disponible en: http: / / scielo.isciii.es/scielo.php?pid=S1135$57272004000300006 \&$ script $=$ sci_arttext\&tlng=en

18. Luengo Matos S, Polo de Santos $M$. Diferencias en los factores relacionados con el uso de la mamografía en las mujeres españolas en los años 1994 y 2000. Rev Esp Salud Pública. 2005;79(5):531-40. Disponible en: http://scielo.isciii.es/scielo. php?pid=S1135-57272005000500003\&script=sci_arttext\&tlng=pt

19. Sankaranarayanan R, Esmy PO, Rajkumar R, Muwonge R, Swaminathan R, Shanthakumari S, et al. Effect of visual screening on cervical cancer incidence and mortality in Tamil Nadu, India: a cluster-randomised trial. The Lancet. 2007;370(9585):398-406. Disponible en: http://www. sciencedirect.com/science/article/pii/S0140673607611957

20. Musre OP. Cáncer de Mama. Epidemiología y Factores de Riesgo. Cuad Méd Soc (Chile). 2007;47(1):1830. Disponible en: http://cms.colegiomedico.cl/ Magazine/2007/47/1/47_1_4.pdf

21. De Charry L, Carrasquilla G, Roca S. Equidad en la detección del cáncer de seno en Colombia. Rev Salud Pública. 2008;10(4):571-82. Disponible en: http://www.scielo.org. $\mathrm{co/pdf/rsap/v10n4/v10n4a07.pdf}$

22. Urrutia MT, Poupin L, Concha X, Viñales D, Iglesias C, Reyes V. ¿ Por qué las mujeres no se toman el Papanicolau?: Barreras percibidas por un grupo de mujeres ingresadas al programa de cáncer cervicouterino AUGE. Revista chilena de obstetricia y ginecología. 2008;73(2):98-103. Disponible en: $\quad$ https://scielo.conicyt.cl/scielo.php?pid=S071775262008000200005 \&script=sci_arttext

23. Peralta MO. Cáncer de mama: estrategias de prevención y vigilancia según nivel de riesgo. Revista Médica Clínica Las Condes. 2011;22(4):436-43.Disponible en: https://www. clinicalascondes.cl/Dev_CLC/media/Imagenes/PDF\%20 revista\%20m\%C3\%A9dica/2011/4\%20julio/3_Dr_Octavio_ Peralta-5.pdf 
Cáncer de cérvix y autorresponsabilidad: perfilando el riesgo de abstención al Papanicolaou entre las mujeres chilenas mediante Árboles de Decisión

24. Kass GV. An exploratory technique for investigating large quantities of categorical data. Applied statistics. 1980;29(2):119-27. Disponible en: http://www.jstor.org/ stable/2986296?seq=1\#page_scan_tab_contents

25. J Han, M Kamber, "Data Mining: Concepts and Techniques San Francisco", Morgan Kaufmann, 2011.

26. Gervilla García E, Palmer Pol A. Predicción del consumo de cocaína en adolescentes mediante árboles de decisión. Revista de Investigación en Educación. 2009;6:7-13.

27. Andreasen AR. Marketing social marketing in the social change marketplace. Journal of Public Policy \& Marketing. 2002;21(1):3-13. Disponible en: http://journals.ama.org/ doi/abs/10.1509/jppm.21.1.3.17602?code=amma-site.
Fuentes de financiamiento:

Este artículo ha sido financiado por los autores.

Conflictos de interés:

Los autores declaran no tener ningún conflicto de interés.

\section{Correspondencia:}

Carlos Rodríguez Garcés

Dirección: Calle 18 de Septiembre 580. Chillán, Chile.

Teléfono: 56-42-2463616

Correo electrónico: carlosro@ubiobio.cl

Recibido: 23 de octubre de 2017

Evaluado: 25 de octubre de 2017

Aprobado: 30 de noviembre de 2017

(c) La revista. Publicado por Universidad de San Martín de Porres, Perú. (c) $\mathbf{\text { Br }}$ Licencia de Creative Commons Artículo en acceso abierto bajo términos de Licencia Creative Commons Atribución 4.0 Internacional. (http://creativecommons.org/licenses/by/4.0/)

ORCID iDs

Carlos Rodríguez Garcés Geraldo Padilla Fuentes

https: / / orcid.org/0000-0002-9346-0780 https: // orcid.org/0000-0003-0882-1818 